\title{
Gender differences in treatment of Coronavirus Disease-2019
}

\author{
Immacolata Ambrosino1 ${ }^{1}$, Elena Barbagelata ${ }^{2}$, Graziamaria Corbi ${ }^{3}$, Tiziana Ciarambino ${ }^{4}$, Cecilia Politi ${ }^{5}$, \\ Anna Maria Morettí \\ ${ }^{1}$ Local Healthcare Unit of Bari, Health District 10, Bari; ${ }^{2}$ Department of Internal Medicine, Lavagna Hospital, Genoa; \\ ${ }^{3}$ Department of Medicine and Health Sciences, University of Molise; Gender Medicine and Aging (GEMEA) Group of \\ the Italian Society of Gerontology and Geriatrics (SIGG); ${ }^{4}$ Department of Internal Medicine, Marcianise Hospital, ASL \\ Caserta; ${ }^{5}$ Head of Gender Medicine Area F.A.D.O.I. (Federazione delle Associazioni dei Dirigenti Ospedalieri

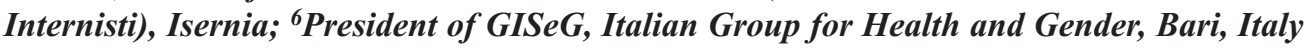

\begin{abstract}
Coronavirus Disease-2019 (COVID-19) is the worst worldwide pandemic with more than $12,000,000$ cases and 560,000 deaths until $14^{\text {th }}$ July 2020 . Men were more infected by COVID19 than women, and male subjects with underlying conditions, including diabetes, hypertension, and cardiovascular diseases developed a severe form of the affection, with increased mortality rate. Many factors can contribute to the disparity in disease outcomes, such as hormone-specific reaction and activity of Xlinked genes, which modulate the innate and adaptive immune response to virus infection. Until now, only the Remdesivir was approved by FDA (Food Drug Administration) for COVID-19 treatment, although several clinical trials are ongoing worldwide also on other drugs. In this review, we analyzed published
\end{abstract}

Correspondence: Immacolata Ambrosino, Local Healthcare Unit of Bari, Health District 10, Corso Benedetto Croce 165, 70125 Bari, Italy.

E-mail: imma-ambrosino@libero.it

Key words: Gender; differences; treatment; COVID-19.

Authors' contributions: IA, study conception and design, revision of all clinical trials, manuscript drafting; EB, TC, revision of all clinical trials, critical revision; GC, CP, critical revision; AMM, study conception and design, critical revision. All authors read and approved the final version of the manuscript.

Conflict of interest: The authors declare that they have no conflicts of interest related to the subject matter or materials discussed in this article.

Received for publication: 14 July 2020.

Accepted for publication: 30 September 2020.

${ }^{\circ}$ Copyright: the Author(s), 2020

Licensee PAGEPress, Italy

Monaldi Archives for Chest Disease 2020; 90:1508

doi: 10.4081/monaldi.2020.1508

This article is distributed under the terms of the Creative Commons Attribution Noncommercial License (by-nc 4.0) which permits any noncommercial use, distribution, and reproduction in any medium, provided the original author(s) and source are credited. studies on several drugs (chloroquine or hydroxychloroquine, remdesivir, favipiravir, lopinavir-ritonavir in combination, tocilizumab, plasma, and immunoglobulins) with some efficacy to COVID-19 in humans, and evaluated if there were a gender analysis of the available data. In our opinion, it is essential to report data about COVID-19 disaggregated by sex, age, and race, because the knowledge of gender differences is fundamental to identify effective and customized treatments to reduce hospitalizations, admissions to intensive care units, and mortality.

\section{Introduction}

Coronavirus disease-2019 (COVID-19) originated in the city of Wuhan and rapidly spread to most countries in the world, so on $12^{\text {th }}$ March 2020 the WHO declared "pandemic" the COVID-19 outbreak [1]. Until $14^{\text {th }}$ July 2020, more than 12 million confirmed cases of COVID-19 and 560 thousand deaths had been reported worldwide [2]. In Italy 243,230 total case of COVID-19 with 34,967 deaths were described [3].

In our previous review, we underlined that, in China and in Italy, men were more infected by COVID-19 than women, most patients were aged 30-79 years, and men with underlying conditions, including diabetes, hypertension and cardiovascular disease developed a severe form of this affection with an increased mortality rate [4]. Many factors can contribute to the disparity in sex-specific disease outcomes. The reduced susceptibility of females to viral infections could be attributed to the protection from $\mathrm{X}$ chromosome and sex hormones, which play an essential role in innate and adaptive immunity. Women may have a lower viral load level than men, a higher number of CD4+ T cells, and higher levels of antibodies. Moreover, the expression of Toll-like receptor 7 (TLR7), encoded by the $\mathrm{X}$ chromosome [5], is higher in women than in men leading to better immune response and viral infection resistance. TLR7 recognizes a single strand RNA virus promoting the production of antibodies against the virus.

Interestingly, in women, the production of pro-inflammatory interleukin-6 (IL-6) after the viral infection is lower than in males and often correlated with better longevity [6].

Severe Acute Respiratory Syndrome Coronavirus 2 (SARSCoV-2) invades human alveolar epithelial cells through angiotensin-converting enzyme 2 (ACE2) [7]. Xie et al. [8] in animal models showed that ACE2 expression dramatically 
reduced with aging in both sexes; also, ACE2 expression appeared to be higher in young than in elderly individuals, and in females than in males, and circulating ACE2 activity was increased in patients with cardiovascular complications [9]. Indeed, the ACE2 gene may be transcriptionally regulated by DNA methylation [10]. The localization of ACE2 on the X chromosome [11] raises the possibility of gender differences in susceptibility and progression of COVID-19 [12]. In particular, theACE2 gene could possibly experience differences in methylation due to X-chromosome activation [13]. Moreover, the male predominance in the COVID-19 pandemic could partially be explained by transmembrane serine protease 2 (TMPRSS2), which could be involved in viral entry and spread in the host. Constitutive expression of TMPRSS2 in lung tissue did not appear to differ between men and women, and low levels of androgens present in women might suffice to sustain TMPRSS2 expression [14].

In our opinion, it is fundamental to report data disaggregated by sex, age, and race, according to the Global Health 50/50 research initiative [15], because the knowledge of gender differences is essential to identify effective and customized treatments to reduce hospitalizations, admissions to intensive care units and mortality.

At the moment, only remdesivir was approved by FDA for COVID-19 treatment, although several clinical trials have been implemented worldwide.

Many drugs, including chloroquine or hydroxychloroquine, remdesivir, favipiravir, lopinavir-ritonavir (used in combination), tocilizumab, plasma, and immunoglobulins, have been highlighted for their promising in vitro results and therapeutic experiences from two other coronavirus diseases - the Severe Acute Respiratory Syndrome and the Middle East Respiratory Syndrome [16]. Several agents have demonstrated some efficacy in human COVID-19 therapy, but mostly through case reports or preliminary data of clinical trials with small sample sizes. Many randomized controlled trials are currently ongoing, to further confirm these results.

The present review aims to investigate the existence of studies evaluating gender differences in the therapeutic response and outcomes in different types of COVID-19 treatment.

\section{Methods}

The included studies were identified by using Pubmed, until $30^{\text {th }}$ June 2020.The search included the following keywords: SARS-CoV-2, COVID-19, gender, sex, chloroquine, hydroxychloroquine, remdesivir, favipiravir, lopinavir-ritonavir, tocilizumab, plasma, and immunoglobulins. We conducted a non-systematic review. Clinical trials, retrospective and prospective studies were included. Studies written in languages other than English were excluded. Three authors (I.A., E.B., and T.C.) reviewed all study abstracts. Studies were included if gender differences in SARS-CoV-2 infection were reported. All selected studies were qualitatively analyzed.

\section{Chloroquine and hydroxychloroquine}

Chloroquine (CQ) and its hydroxyl analogue hydroxychloroquine (HCQ), a widely-used antimalarial and autoimmune dis- ease drug, are prominent on the list of potential COVID-19 treatments.

Chloroquine is known to block virus infection by increasing endosomal $\mathrm{pH}$ required for virus/cell fusion, as well as by interfering with the glycosylation of cellular receptors of SARSCoV-2 [17]. Wang et al. demonstrated that chloroquine functioned at both entry and post-entry stages of the 2019-nCoV infection in vitro [18]. Moreover, Yao et al. [19] evidenced that HCQ was more potent than CQ in vitro.

Besides its antiviral capability, CQ has an immune-modulating activity, which may synergistically enhance its antiviral effect in vivo. After oral administration, CQ distributed in the whole body, including lung. The effectiveness of HCQ for treating COVID-19 is the object of several studies.

In an observational study published on New England Journal of Medicine [20] (Table 1), a large sample of consecutive patients $(n=1446)$, hospitalized with COVID-19 between $7^{\text {th }}$ March and $8^{\text {th }}$ April 2020, was recruited. Of 1376 included patients, $811(58.9 \%$ ) received HCQ (with a median duration of treatment of 5 days), and 565 (41.1\%) did not. $45.8 \%$ received HCQ in the 24 hours between their presentation to the emergency department and the start of study follow-up, and $85.9 \%$ received the treatment within 48 hours after admission to the emergency department. In the unmatched sample, HCQ exposure differed according to age group, sex, race and ethnic group, body-mass index, insurance, smoking status, and current use of other medications. Hydroxychloroquine-treated patients had a lower $\mathrm{PaO}_{2}: \mathrm{FIO}_{2}$ at baseline than patients who did not receive HCQ.

In this analysis, hydroxychloroquine administration was not associated with either a greatly lowered or an increased risk of the composite endpoint of intubation or death, but no gender analysis was available.

Tang et al. [21] (Table 1) conducted a multicentre randomized open-label trial in which patients with COVID-19, admitted from $11^{\text {th }}$ to $29^{\text {th }}$ February 2020 to the hospitals of three provinces in China (Hubei, Henan, and Anhui), were enrolled, stratified according to disease severity (mild/moderate or severe), and randomised into treatment (hydroxychloroquine plus standard of care) and control (standard of care only) group.

Of 150 randomised patients, 75 patients were assigned to standard care (SOC) and 75 patients to SOC plus HCQ at a loading dose of $1200 \mathrm{mg}$ daily for 3 days, followed by a maintenance dose of $800 \mathrm{mg}$ daily for the remaining days (SOC plus HCQ). The trial showed that among 150 patients, 82 (55\%) were male of whom $42(56 \%)$ were in the SOC plus HCQ group, while 40 $(53 \%)$ were in the SOC group. The mean age of the patients was 46 years, of whom 48 years in the SOC plus HCQ group, and 40 years in the SOC group. Gender analysis was not carried out in this trial. The study concluded that the administration of hydroxychloroquine did not result in a significantly higher probability of negative conversion than SOC alone in patients admitted to hospital with mainly persistent mild to moderate COVID-19, and the number of adverse events was higher in hydroxychloroquine recipients than in non-recipients.

In the observational study of Mahévas et al. [22] (Table 1) two groups (treatment group with HCQ at $600 \mathrm{mg} /$ day and control group with no HCQ treatment) were identified, through the electronic health records' examine of all patients with COVID19 pneumonia, who required oxygen but not intensive care, and admitted to four French tertiary hospitals between $12^{\text {th }}$ and $31^{\text {st }}$ March 2020. The study showed a median age of patients of 60 years, $72 \%$ of them were men. The HCQ treatment at 600 
$\mathrm{mg}$ /day added to SOC was not associated with a reduction of admissions to the intensive care unit or death, 21 days after hospital admission, compared with SOC alone. Also, the rate of survival without acute respiratory distress syndrome did not increase. The results of this study did not support its use in patients admitted to the hospital with COVID-19 requiring oxygen. This study did not reveal differences in response to therapy in the two sexes, in different age groups or related to different comorbidities.

In a retrospective multicenter cohort study [23] (Table 1) from a sample of 7914 patients with COVID-19 admitted in New York metropolitan hospitals during March $15^{\text {th }}$ through $28^{\text {th }}$, a total of 2362 records were randomly selected, and 1438 were abstracted and included in the analyses. Of these patients, $735(51.1 \%)$ received $\mathrm{HCQ}+$ azithromycin, 271 (18.8\%) received HCQ alone, 211 (14.7\%) received azithromycin alone, and $221(15.4 \%)$ received neither drug.

Black or Hispanic patients were as likely to receive HCQ and/or azithromycin. Median patient age was similar in the 4 groups (HCQ+azithromycin, 61.4 years; HCQ alone, 65.5 years; azithromycin alone, 62.5 years; and neither drug, 64.0 years). Patients receiving $\mathrm{HCQ}+$ azithromycin and HCQ alone were more likely to be obese and have diabetes than those in the groups receiving azithromycin alone and neither drug. Patients receiving alone had the highest levels of chronic lung disease $(25.1 \%)$ and cardiovascular conditions (36.5\%). Among patients hospitalized in metropolitan New York with COVID-19, treatment with HCQ, azithromycin, or both, compared with neither treatment, was not significantly associated with differences in in-hospital mortality. Patients who received hydroxychloroquine with or without azithromycin were more likely (relative to patients receiving neither drug) to be male (hydroxychloroquine +azithromycin, 62\%; hydroxychloroquine alone, 58,3\%; azithromycin alone, 63,5\%; and neither drug, 49,8\%), have preexisting medical conditions, and have impaired respiratory or liver function at presentation. There were no significant differences in mortality between inpatients who received hydroxychloroquine with or without azithromycin and inpatients who received neither drug.

The findings of this study also confirm what other studies shown about the natural history of COVID-19 infection in the US: poor hospital outcomes were associated with male sex; preexisting conditions such as hypertension, obesity, and diabetes; and presenting findings such as elevated liver enzymes and abnormal kidney function. There was no evidence in this study that black or Hispanic persons were prescribed these medications at a lower rate than white patients, which is relevant given the population-level differences in COVID-19 deaths previously reported by race and ethnicity.

In the open-label non-randomized clinical trial conducted by Gautret et al. [24] (Table 1), 26 French confirmed positive COVID-19 patients were included in a single-arm protocol, from early March to March $16^{\text {th }}$, to receive $600 \mathrm{mg}$ of hydroxychloroquine daily. Their viral load in nasopharyngeal swabs was tested daily in a hospital setting. A control group included 16 patients. Among hydroxychloroquine-treated patients, six subjects received azithromycin $(500 \mathrm{mg}$ on day 1 followed by 25 $0 \mathrm{mg}$ per day, the next four days) to prevent bacterial super-infection under daily electrocardiogram control. Overall, 15 patients were male $(41.7 \%)$, with a mean age of 45.1 years. No significant difference was observed between hydroxychloroquinetreated and control patients concerning gender, clinical status, and duration of symptoms before the study inclusion. In this trial, there was no analysis of different age groups and comorbidities. At day 6 post-inclusion, $100 \%$ of patients treated with hydroxychloroquine and azithromycin combination were virologically cured, compared with $57.1 \%$ of patients treated with hydroxychloroquine only, and $12.5 \%$ of patients who did not receive hydroxychloroquine $(\mathrm{p}<0.001)$. Despite its small sample size, this survey showed that hydroxychloroquine treatment is significantly associated with viral load reduction/disappearance in COVID-19 patients, and its effect is reinforced by azithromycin.

In contrast with the previous study, Molina et al. [25] (Table 1 ), in their prospective study, concluded that there was no evidence of rapid antiviral clearance or clinical benefit with the combination of HCQ and azithromycin in patients with severe COVID-19 infection. In their small study including 11 consecutive in-patients who received hydroxychloroquine $(600 \mathrm{mg} / \mathrm{d}$ for 10 days) and azithromycin (500 $\mathrm{mg}$ day 1 and $250 \mathrm{mg}$ days 2 to 5 ), using the same dosing regimen reported by Gautret et al. [24], there were 7 men and 4 women with a mean age of 58.7 years (range: 20-77), and 8 had significant comorbidities associated with poor outcomes (obesity: 2; solid cancer: 3 ; hematological cancer: 2; HIV-infection: 1).

Also, in the study conducted by Chen et al. [26] (Table 1) 30 treatment-naïve patients with confirmed COVID-19 were randomized 1:1 to HCQ group (HCQ $400 \mathrm{mg}$ per day for 5 days plus conventional treatments) and the control group (conventional treatment only). The authors did not found a difference in the rate of virologic clearance at 7 days, with or without 5 daystreatment of hydroxychloroquine, and no difference in duration of hospitalization, temperature normalization, and radiological progression. Again, no gender analysis was performed. In the randomized clinical trial of Chen et al. [27] (Table 1) since $4^{\text {th }}$ to $28^{\text {th }}$ February 2020, 62 COVID-19 patients admitted to Renmin Hospital of Wuhan University were randomized in two groups. All received the standard treatment (oxygen therapy, antiviral agents, antibacterial agents, and immunoglobulin, with or without corticosteroids), patients in the HCQ treatment group received additional oral HCQ $400 \mathrm{mg} / \mathrm{d}$ (200 mg/bid) between days 1 and 5, patients in the control group only the standard treatment. The total population included $46.8 \%$ (29 of 62 ) of male and $53.2 \%$ of (33 of 62 ) female, the mean age was 44.7 years. The time to clinical recovery (TTCR), the body temperature recovery time and the cough remission time were significantly shortened in the HCQ treatment group. The study concluded that among patients with COVID-19, the use of HCQ could significantly shorten TTCR and promote the absorption of pneumonia. In this study, no difference in the age and sex distribution between the control group and the HCQ group was observed.

Borba et al. [28,29] (Table 1) conducted a parallel doublemasked randomized, phase IIb clinical trial with 81 adult patients hospitalized with SARS-CoV-2 infection since $23^{\text {rd }}$

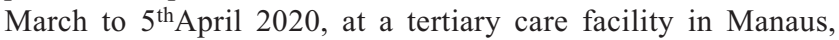
Brazilian Amazon. All randomized patients were allocated to receive high-dosage HCQ (i.e., $600 \mathrm{mg}$ CQ twice daily for 10 days) (41 [50.6\%] high-dosage group) or low-dosage CQ (i.e., $450 \mathrm{mg}$ twice daily on day 1 and once daily for 4 days) (40 [49.4\%] low-dosage group). The enrolled patients had an overall mean (SD) age of 51.1 (13.9) years and a predominance of men (60 [75.3\%]). Hypertension (25 of 55 [45.5\%]), alcohol use disorder (14 of 51 [27.5\%]), and diabetes (14 of 55 [25.5\%]) were the most frequent comorbidities. Older age (mean [SD] age, 54.7 [13.7] years vs 47.4 [13.3] years), and a higher rate of heart dis- 
ease (5 of 28 [17.9\%] vs 0) was found in the high-dose group. The preliminary findings of this study suggest that the higher CQ dosage should not be recommended for critically ill patients with COVID-19, because of its potential safety hazards, especially when taken concurrently with azithromycin and oseltamivir. These findings cannot be extrapolated for patients with non-severe COVID-19. Aging might be associated with unfavorable outcomes.

\section{Remdesivir}

Remdesivir (also GS-5734) is a monophosphoramidate prodrug of an adenosine analogue that has a broad antiviral spectrum including filoviruses, paramyxoviruses, pneumoviruses, and coronaviruses [30].

On $3^{\text {th }}$ July 2020 this drug was approved by the EMA for the treatment of COVID-19 in adults and adolescents. In vitro, remdesivir inhibits all human and animal coronaviruses including SARS-CoV-2 [30,31]. Remdesivir is a potent inhibitor of SARS-CoV-2 replication in human nasal and bronchial airway epithelial cells [32]. Recently, remdesivir was regarded as promising for COVID-19 therapy because case studies have reported some benefit in severely ill patients with COVID-19, but interim published results are disappointing. Intravenous remdesivir has been used based on individual compassionate use over the past several months in patients with COVID-19 in some countries [33] (Table 1); in particular 22 patients were enrolled in the United States, 9 in Japan, 22 in Europe, and 1 in Canada.75\% of patients were men, the age range was 23 to 82 years, and the median age was 64 years.

In the placebo-controlled randomised trial by Wang et al. [34] (Table 1), conducted to assess the effectiveness and safety of intravenous remdesivir in patients with severe COVID-19 admitted to hospital in Wuhan, 237 patients with pneumonia were enrolled, 158 patients were assigned to receive remdesivir and 79 to receive the placebo; sex distribution was $89(56 \%)$ men versus $69(44 \%)$ women in the remdesivir group and 51 (65\%) versus 27 (35\%) in the placebo group. The median age of study patients was 65 years. Remdesivir was adequately tolerated but did not provide significant clinical or antiviral effects in seriously ill patients with COVID-19 [34]. However, there was no gender analysis, so it is not possible to exclude clinically meaningful differences between men and women, and numerical reductions in some clinical parameters.

In the preliminary report of Beigel et al. [35] (Table 1) intravenous remdesivir was superior to placebo in shortening the time to recovery in adults hospitalized with COVID-19, in reducing evidence of lower respiratory tract infection; also in this trial male sex was more represented $(64.3 \%$ of all patients were men), but sex-specific data on adverse reactions or appropriate dose adjustments are missing.

\section{Lopinavir, favipiravir, ritonavir}

Lopinavir is a human immunodeficiency virus (HIV) type 1 aspartate protease inhibitor with in vitro inhibitory activity against SARS-CoV, the virus that causes SARS in humans [36]. Similarly, lopinavir has shown activity, both in vitro and in an animal model [37] against Middle East respiratory syndrome coronavirus (MERS-CoV), and case reports have suggested that the combination of lopinavir-ritonavir with ribavirin and interferon alfa resulted in virologic clearance and survival [38]. In hospitalized adult patients with severe COVID-19, no benefit was observed with lopinavir-ritonavir treatment. In the randomized, controlled, open-label trial involving hospitalized adult patients with confirmed SARS-CoV-2 infection, $60.3 \%$ of the 199 patients who underwent randomization were men but also in this trial lack sex-specific data on adverse reactions or appropriate dose adjustments [39] (Table 1). Similar outcomes have been seen for favipiravir and ritonavir, with the latter causing severe side effects.

There is evidence that women encounter more often adverse drug reactions to antiviral treatment than men. Also, pharmacokinetics and treatment responses to antiretroviral therapy with ritonavir and lopinavir differ between males and females [40] Higher plasma concentrations of ritonavir and a higher total cholesterol:high-density lipoprotein (HDL) ratio have been reported in young females [41], while an atazanavir plus ritonavir regimen was associated with higher risk of virologic failure in women as compared to men [42].

As long as drug trials continue to enroll both men and women but fail to sex-disaggregated outcome data, costly mistakes will continue.

\section{Tocilizumab}

Tocilizumab (TCZ), is a recombinant humanized anti-human monoclonal antibody of the immunoglobulin $\mathrm{G} 1_{\mathrm{k}}$ subclass, directed against soluble and membrane-bound interleukin 6 receptors [43]. TCZ was firstly approved in 2005 as an orphan drug in Japan for the therapy of Castleman's disease [44]. TCZ is used in the EU, alone or in combination with anti-rheumatic drugs (DMARDs), to treat adults with moderate to severely active rheumatoid arthritis (RA), and over-2-years children with the systemic or polyarticular form of juvenile idiopathic arthritis [45]. TCZ inhibits the binding of IL-6 to its receptors, so it reduces this cytokine's pro-inflammatory activity by competing with both the soluble and membrane-bound forms of the human IL-6 receptor (IL-6R) [46]. It is recommended in seriously ill patients with elevated IL-6 for the treatment of pneumonia by COVID-19.

In one study (Table 1) that enrolled 15 patients (12 males and 3 females) with Covid-19, the authors described that $13 \%$ of patients were moderately ill, $40 \%$ were seriously ill and $47 \%$ were critically ill. However, they describe that only 1 patient (a 80-year-old female) had a clinical outcome of aggravation [47]. In another study (Table 1) that enrolled 21 patients, with mean age $56 \pm 16$ years-old ( 18 male and 3 female), the authors reported that within 5 days after TCZ therapy, $75 \%$ of patients had lowered their oxygen intake, and 1 patient needed no oxygen therapy [48]. Unfortunately, no data described in literature gender differences in course of TCZ treatment. No real-life data on the effect by gender of TCZ on the inflammatory activity in COVID-19 patients. 


\section{Convalescent plasma/immunoglobulins}

Convalescent plasma, convalescent serum and hyperimmune immunoglobulin prepared from convalescent plasma, are interventions used in the past to treat conditions when no vaccine or pharmacological interventions were available. It has been reported that the use of convalescent plasma may reduce mortality, appears safe, and the effectiveness of convalescent plasma in reducing hospital length of stay is dependent on early administration of the therapy, and in prophylaxis is more likely to be beneficial than in treating severe disease [49]. Plasma transfusions are also known to cause transfusion-associated circulatory overload (TACO). TACO is especially important to consider, because COVID-19 patients with comorbidities likely eligible for experimental treatment with convalescent plasma therapy, are at an increased risk of these adverse events. Besides, to the mentioned adverse events, transfusion-transmitted infections, red blood cell alloimmunization and hemolytic transfusion reactions have also been described following plasma transfusion, although they are less common [50]. Shen et al. reported that convalescent plasma could be a treatment option for COVID-19 patients with respiratory failure [51] (Table 1), and the authors reported that this procedure improved the clinical situation, decreased the patient's viral load, and induced a negativization of patients within 12 days after the transfusion [51]. One limitation of these data is that all patients received antiviral medications and steroids before receiving their convalescent plasma. Another study showed that 2 elderly (1 male and 1 female) patients improved after the application of convalescent plasma [52] (Table 1). No studies reported specifically data on the gender differences in the convalescent plasma treatment.

Immunoglobulin therapy has been used for the prevention and treatment of infectious diseases before the introduction of antimicrobial agents into clinical practice. In the early 1890 s, Emil von Behring and Shibasaburo Kitasato set the basis of "serum therapy" showing that antibody preparations derived from the serum of immunized animals have the ability to protect against bacterial toxins [53]. In the pre-antibiotic era, serum therapy significantly reduced the mortality in some infectious such as meningococcal and Haemophilus influenzae meningitis, pneumococcal pneumonia, and diphtheria. The efficacy of serum therapy varied with the type and severity of the infections and the timing of treatment administration in relation to symptom onset [54]. The use of immunoglobulins for the infectious diseases can involve the passive transfer of antibodies for pre/post exposure prophylaxis or for treatment. Passive immunization provides temporal immunity to un-immunized individuals either prophylactically or therapeutically [55]. In particular, immunology clearly proves that antibodies in the blood or in the plasma fraction of the blood recognize epitopes on pathogens (e.g., viruses). They either neutralize them or reduce the virus load in conjunction with cellular responses to prevent or eventually cure the disease. Thus, antibodies are very efficient endogenous molecules that initiate and carry out self-healing processes in the human body [56]. Few data are reported on the gender differences in Immunoglobulins therapy. In particular, it has been described that in the severe status more female patients had a high level of $\operatorname{IgG}$ antibody compared to male patients, and the production of $\operatorname{IgG}$ antibody tended to be stronger in female patients in the early phase of COVID-19 [57]. This study analyzed 304 patients (204 female), and the authors demonstrated that, in the severe status, the SARS-CoV-2 IgG antibody, in females, was more than $100 \mathrm{AU} / \mathrm{mL}$, while in males, the $\mathrm{IgG}$ antibody was under $100 \mathrm{AU} / \mathrm{ml}$. These data suggest that more female patients generate a high level of SARS-CoV-2 IgG antibody in comparison to male patients, in the severe status of the COVID-19 infection. However, the result showed that the concentration of the SARS-CoV-2 IgG antibody in female patients tended to be higher than male patients in 2 to 4 weeks after disease onset, and the difference in antibody concentration disappeared after 4 weeks of disease onset [57]. Therefore, the authors propose that more attention should be paid to the patients whose $\mathrm{IgG}$ antibody was at low levels, and monitoring the IgG antibody may be a potential method to predict COVID19 prognosis.

Adverse reactions after immunoglobulins treatment have been reported. In particular, it has been described that the dermatological disorders are more frequent in male patients [58], and that donations from female donors, especially those with a history of pregnancy, can induce an adverse reaction in male patients. No studies reported specifically data on the gender differences in the convalescent plasma treatment.

\section{Conclusions}

In the current state of knowledge, only remdesivir was approved by FDA for COVID-19 treatment. Some antiviral drugs have been tested in randomized clinical trials, but there has not been explicit consideration of sex biases in drug efficacy or adverse (potentially lethal) reactions. This occurs despite previous studies showing clear and important differences in drug treatment responses, including antivirals. Drug trials are yet designed and analyzed without appropriate attention toward sexspecific dosages or differential side effects. Currently, there are over 1000 registered trials on COVID-19 treatment, more than half of which include pharmacologic intervention or observation, and seven completed trials [59]. All trials to date include both men and women, but take a sex-blind approach to the analyses of outcome data, with no governmental guidelines mentioning sex-specific prophylactic or therapeutic recommendations (except for pregnant and postpartum women).

In our review, we analyzed clinical trials, which evaluated several drugs such as hydroxychloroquine, remdesivir, favipiravir, lopinavir-ritonavir (used in combination), tocilizumab, plasma, and immunoglobulins, in patients with COVID-19. In all the study we did not find COVID-19 disaggregated by sex, age, and race data related to hospitalizations, admissions to intensive care units, and mortality.

In our opinion, these data are essential to evaluate the effectiveness of a drug in men and women, in different age groups and race groups as well as the appearance of side effects and the outcomes. Every patient is different, so we cannot think to treat everyone in the same way. For this reason, it is crucial to collect and analyzed all gender data to test and identify specific therapies for each patient. It is necessary to provide personalized therapy to patients with COVID-19 because using the right therapy to the right patient in the early stage of disease will allow to obtain a reduction of hospitalizations, admissions to intensive care units, and mortality. In conclusion in our opinion supporting gender analysis and sex-disaggregated data is an integral part of a strong and successful COVID-19 response. 


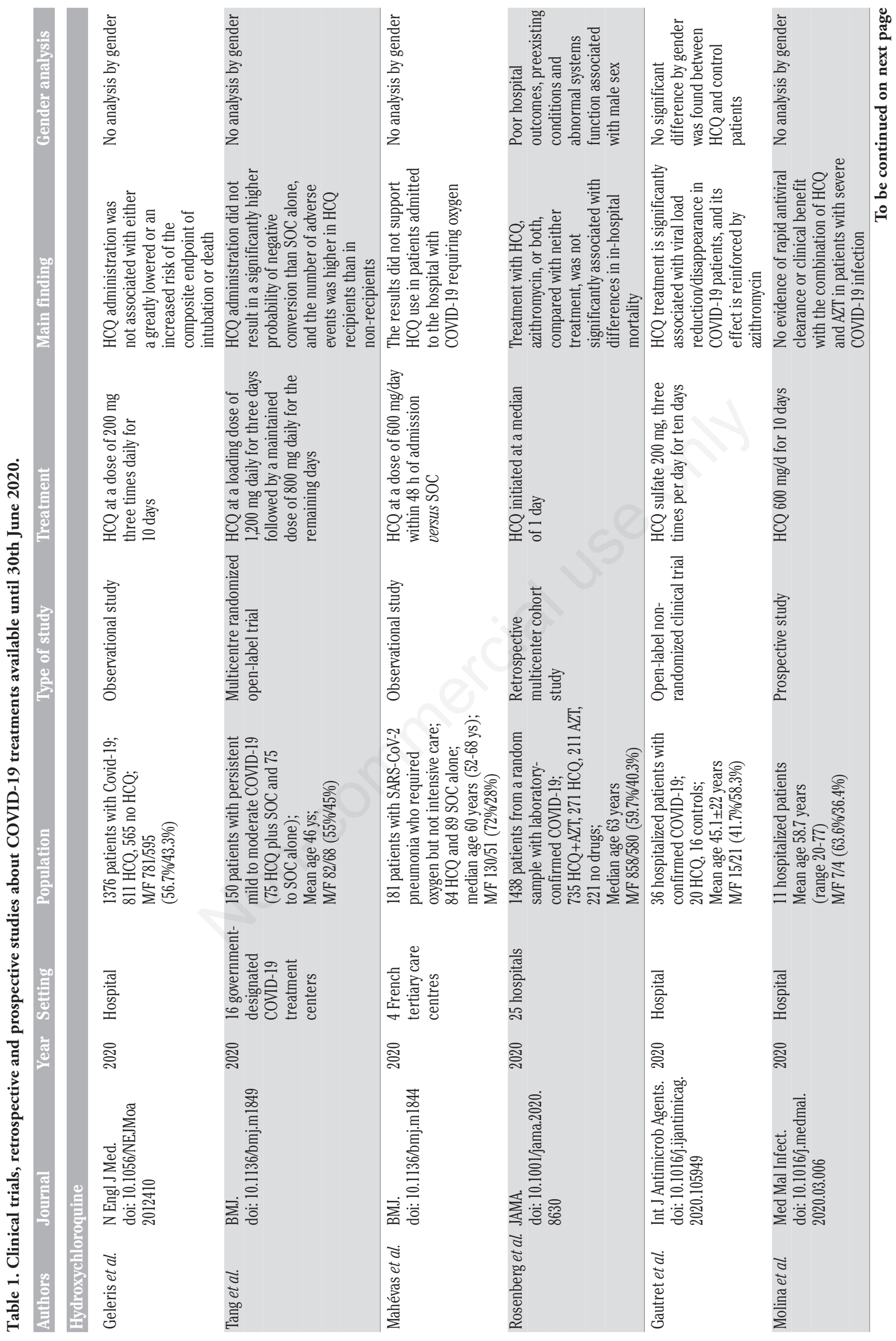




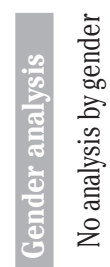
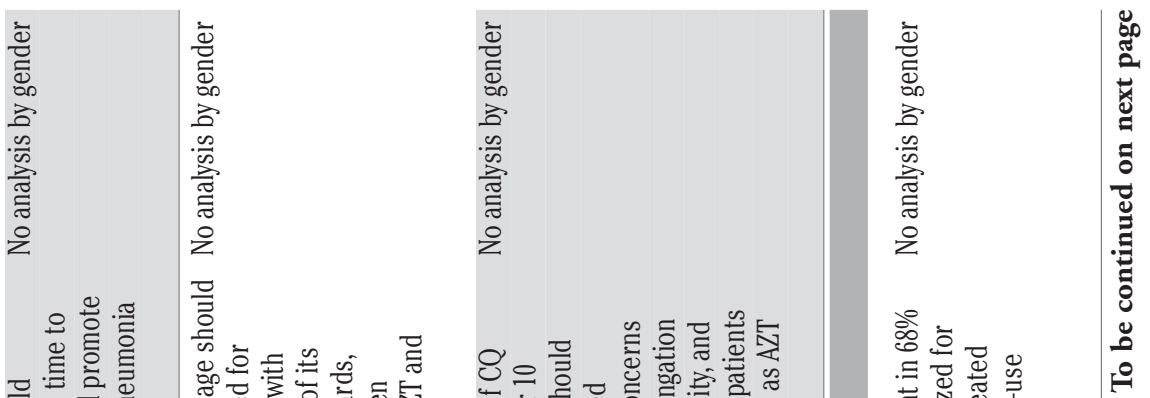

“

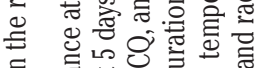
들 항 휸흔

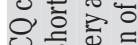

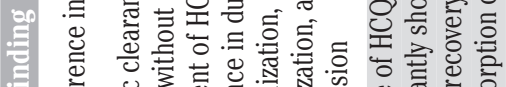

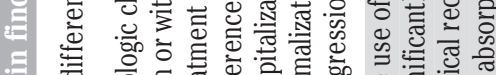

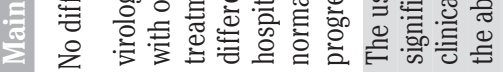

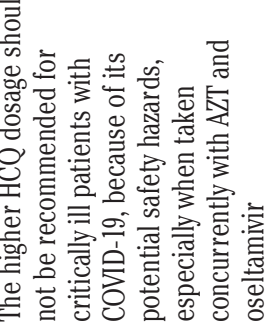

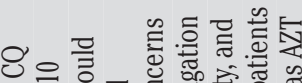

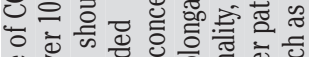

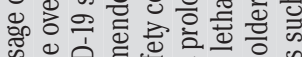

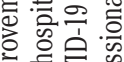

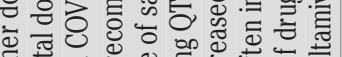

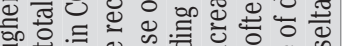

on

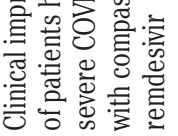

崖

屁哭导

选造

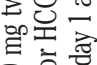

言

幽

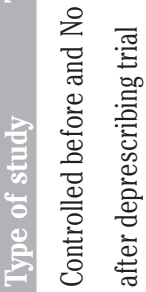

鹿

窵

离的高

责芯怘这

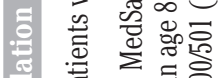

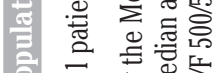

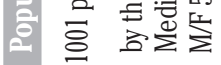

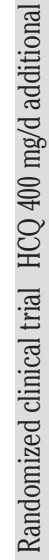

容客

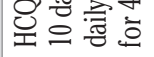

总會

党急

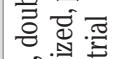

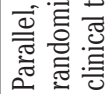

๘

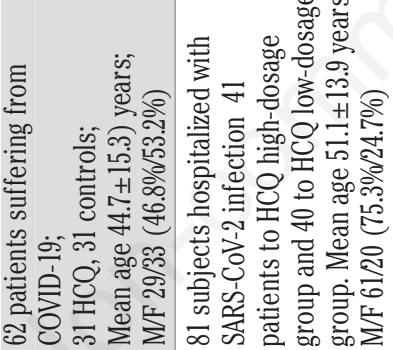

호웜

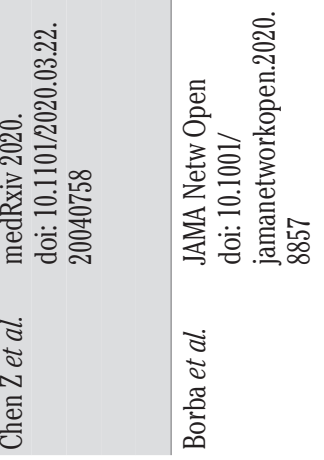

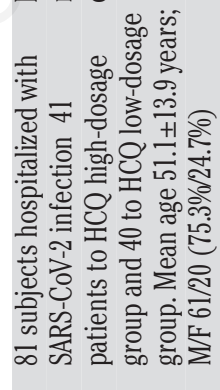

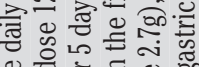

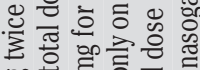

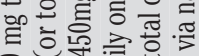

응 웡워

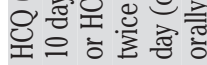

気。

票

离

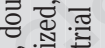

홇

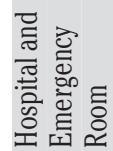

ลิ

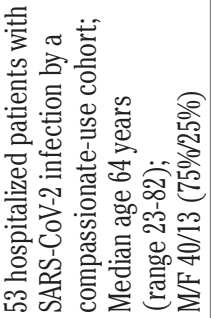

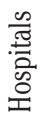

ลั

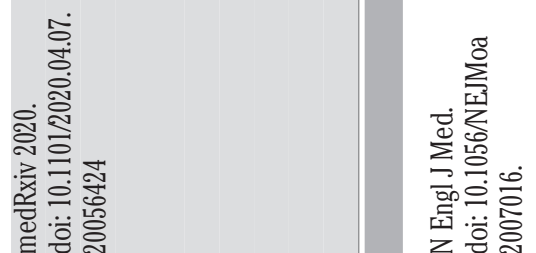

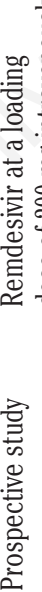

궁

on

.

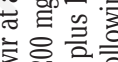

की

要

ฯ 응 


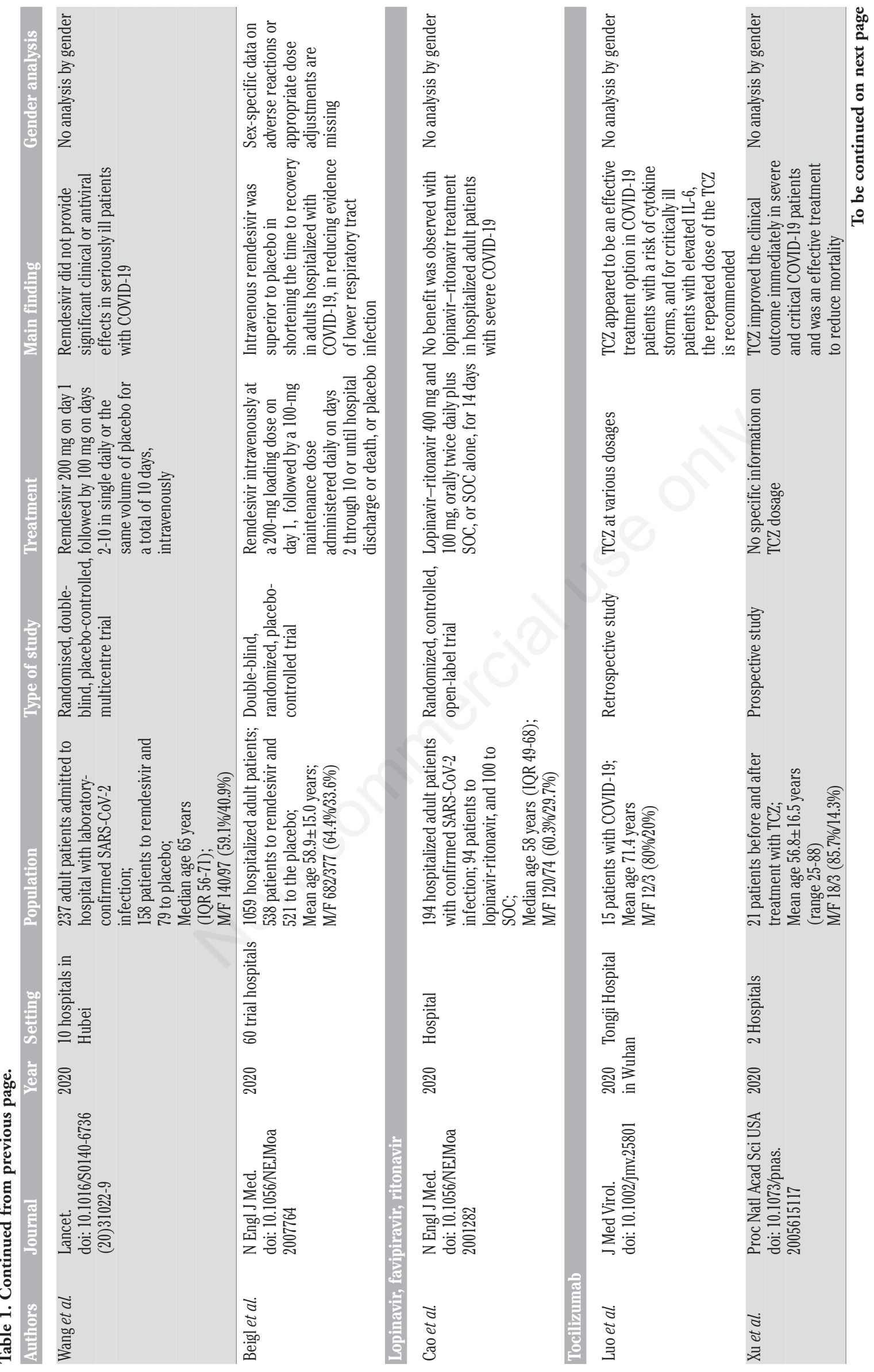




\section{References}

1. WHO. Clinical management of severe acute respiratory infection (SARI) when COVID-19 disease is suspected. Available from: https://www.who.int/publications-detail/clinical-management-of-severe-acute-respiratory-infection-when-novelcoronavirus-(ncov)-infection-is-suspected

2. WHO. WHO Coronavirus Disease (COVID-19) Dashboard. Available from: https://who.sprinklr.com/

3. Ministero della Salute. Nuovo Coronavirus. Available from: http://www.salute.gov.it/portale/nuovocoronavirus/homeNuov oCoronavirus.jsp

4. Ambrosino I, Barbagelata E, Ortona E, et al. Gender differences in patients with COVID-19: a narrative review. Monaldi Arch Chest Dis 2020;90:1389. doi: 10.4081/monaldi. 2020.1389

5. de Groot NG, Bontrop RE. COVID-19 pandemic: is a genderdefined dosage effect responsible for the high mortality rate among males? Immunogenetics 2020;72:275-7.

6. Zhu Z, Cai T, Fan L, et al. Clinical value of immune-inflammatory parameters to assess the severity of coronavirus disease 2019. Int J Infect Dis 2020;95:332-9.

7. Zhou P, Yang X-L, Wang X-G, et al. A pneumonia outbreak associated with a new coronavirus of probable bat origin. Nature 2020;579:270-3.

8. Xie X, Xudong X, Chen J, et al. Age- and gender-related difference of ACE2 expression in rat lung. Life Sci 2006;78: 2166-71.

9. Soro-Paavonen A, Gordin D, Forsblom C, et al. Circulating ACE2 activity is increased in patients with type 1 diabetes and vascular complications. J Hypertens 2012;30:375-83.

10. Zill P, Baghai TC, Schule C, et al. DNA methylation analysisof the angiotensin converting enzyme (ACE) gene in majordepression. PLoS One 2012;7:e40479.

11. Tipnis SR, Hooper NM, Hyde R, et al. A human homolog of angiotensin-converting enzyme. Cloning and functional expression as a captopril-insensitive carboxypeptidase. J Biol Chem 2000;275:33238-43.

12. Jin J-M, Bai P, He W, et al. Gender differences in patients with COVID-19: focus on severity and mortality. Front Public Health 2020;8:152.

13. Perrotta F, Corbi G, Mazzeo G, et al. COVID-19 and the elderly: insights into pathogenesis and clinical decision-making. Aging Clin Exp Res 2020. doi: 10.1007/s40520-020-01631-y

14. Stopsack KH, Mucci LA, Antonarakis ES, et al. TMPRSS2 and COVID-19: Serendipity or opportunity for intervention? Cancer Discov 2020;10:779-82.

15. Global Health 5050 [Internet]. Sex, gender and COVID-19. Accessed on: 11 April 2020. Available from: https://globalhealth5050.org/covid19/

16. Dyall J, Gross R, Kindrachuk J, et al. Middle East respiratory syndrome and severe acute respiratory syndrome: current therapeutic options and potential targets for novel therapies. Drugs 2017;77:1935-66. doi: 10.1007/s40265-017-0830-1

17. Vincent MJ, Bergeron E, Benjannet S, et al. Cloroquine is a potent inhibitor of SARS coronavirus infection and spread. Virol J 2005;2:69. doi: 10.1186/1743-422X-2-69

18. Wang M, Cao R, Zhang L, et al. Remdesivir and chloroquine effectively inhibit the recently emerged novel coronavirus (2019-nCoV) in vitro. Cell Res 2020;30:269-71. doi: 10.1038/s41422-020-0282-0

19. Yao X, Ye F, Zhang M, et al. In vitro antiviral activity and pro- 
jection of optimized dosing design of hydroxychloroquine for the treatment of severe acute respiratory syndrome coronavirus 2 (SARS-CoV-2). Clin Infect Dis 2020;71:732-9. doi: $10.1093 / \mathrm{cid} / \mathrm{ciaa} 237$

20. Geleris J, Sun Y, Platt J et al. Observational study of hydroxychloroquine in hospitalized patients with Covid-19. N Engl J Med 2020;382:2411-8. doi: 10.1056/NEJMoa2012410

21. Tang W, Cao Z, Han M, et al. Hydroxychloroquine in patients with mainly mild to moderate coronavirus disease 2019: open label, randomised controlled trial. BMJ 2020;369:m1849. doi: 10.1136/bmj.m1849

22. Mahévas M, Tran VT, Roumier M, et al. Clinical efficacy of hydroxychloroquine in patients with covid-19 pneumonia who require oxygen: observational comparative study using routine care data. BMJ 2020;369:m1844. doi:10.1136/bmj.m1844

23. Rosenberg ES, Dufort EM, Udo T, et al. Association of treatment with hydroxychloroquine or azithromycin with in-hospital mortality in patients with COVID-19 in New York State. JAMA 2020;323:2493-502. doi:10.1001/jama.2020.8630

24. Gautret P, Lagier JC, Parola P, et al. Hydroxychloroquine and azithromycin as a treatment of COVID-19: results of an open-label non-randomized clinical trial. Int J Antimicrob Agents 2020;56:105949. doi: 10.1016/j.ijantimicag. 2020 . 105949

25. Molina JM, Delaugerre C, Le Goff J, et al. No evidence of rapid antiviral clearance or clinical benefit with the combination of hydroxychloroquine and azithromycin in patients with severe COVID-19 infection. Med Mal Infect 2020;50:384. doi: 10.1016/j.medmal.2020.03.006

26. Chen J, Liu D, Lui L, et al. A pilot study of hydroxychloroquine in treatment of patients with moderate COVID-19. Zhejiang Da Xue Xue Bao Yi Xue Ban 2020;49:215-9. doi: 10.3785/j.issn.1008-9292.2020.03.03

27. Chen Z, Hi J, Zhang Z, et al. Efficacy of hydroxychloroquine in patients with COVID-19: results of a randomized clinical trial. medRxiv 2020. doi: 10.1101/2020.03.22.20040758

28. Borba MGS, Val FFA, Sampaio VS, et al. Effect of high vs low doses of chloroquine diphosphate as adjunctive therapy for patients hospitalized with Severe Acute Respiratory Syndrome Coronavirus 2 (SARS-CoV-2) infection: A randomized clinical trial. JAMA Netw Open 2020;3:e208857. doi: 10.1001/ jamanetworkopen.2020.8857

29. Borba MGS, de Almeida Val F, Sampaio VS, et al. Chloroquine diphosphate in two different dosages as adjunctive therapy of hospitalized patients with severe respiratory syndrome in the context of coronavirus (SARS-CoV-2) infection: Preliminary safety results of a randomized, double-blinded, phase IIb clinical trial (CloroCovid-19 Study). medRxiv 2020. doi: 10.1101/2020.04.07.20056424

30. Sheahan TP, Sims AC, Graham RL, et al. Broad-spectrum antiviral GS-5734 inhibits both epidemic and zoonotic coronaviruses. Sci Transl Med 2017;9:eaal3653.

31. Brown AJ, Won JJ, Graham RL, et al. Broad spectrum antiviral remdesivir inhibits human endemic and zoonotic deltacoronaviruses with a highly divergent RNA dependent RNA polymerase. Antiviral Res 2019;169:104541.

32. Pizzorno A, Padey B, Julien T, et al. Characterization and treatment of SARS-CoV-2 in nasal and bronchial human airway epithelia. bioRxiv 2020. doi: 10.1101/2020.03.31.017889

33. Grein J, Ohmagari N, Shin D, et al. Compassionate use of remdesivir for patients with severe Covid-19. N Engl J Med 2020;382:2327-36. doi: 10.1056/NEJMoa2007016

34. Wang Y,Zang D, Du G, et al. Remdesivir in adults with severe
COVID-19: a randomized, double-blind, placebo-controlled, multicentre trial. Lancet 2020;395:1569-78. doi: 10.1016/ S0140-6736(20)31022-9

35. Beigl JH, Tomashek KM, Dodd LE et al. Remdesivir for the treatment of Covid-19 - Preliminary report. N Engl J Med 2020;389:992-4. doi: 10.1056/NEJMoa2007764

36. Chu CM, Cheng VC, Hung IF, et al. Role of lopinavir/ritonavir in the treatment of SARS: initial virological and clinical findings. Thorax 2004;59:252-6.

37. Chan JF-W, Yao Y, Yeung M-L, et al. Treatment with lopinavir/ritonavir or interferon- $\beta 1 \mathrm{~b}$ improves outcome of MERS-CoV infection in a nonhuman primate model of common marmoset. J Infect Dis 2015;212:1904-13.

38. Kim UJ, Won E-J, Kee S-J, et al. Combination therapy with lopinavir / ritonavir, ribavirin and interferon- $\alpha$ for Middle East respiratory syndrome. Antivir Ther 2016;21:455-9.

39. Cao B, Wang Y, Wen D, et al. A trial of lopinavir-ritonavir in adults hospitalized with severe COVID-19. N Engl J Med 2020; 382:1787-99. doi: 10.1056/NEJMoa2001282

40. Shiau S, Kuhn L, Strehlau R, et al. Sex differences in responses to antiretroviral treatment in South African Hiv-infected children on ritonavir-boosted lopinavir- and nevirapine-based treatment. BMC Pediatr 2014; 14:39.

41. Umeh OC, Currier JS, Park JG, et al. Sex differences in lopinavir and ritonavir pharmacokinetics among Hiv-infected women and men. J Clin Pharmacol 2011;51:1665-73.

42. Smith KY, Tierney C, Mollan K, et al. Outcomes by sex following treatment initiation with atazanavir plus ritonavir or efavirenz with abacavir/lamivudine or tenofovir/emtricitabine. Clin Infect Dis 2014;58:555-63.

43. European Medicines Agency [Internet]. Assessment Report ForRoActemra. Available from: https:/www.ema.europa.eu/en/ medicines/human/EPAR/roactemra

44. Higuchi T, Nakanishi T, Takada K, et al. A case of multicentric Castleman's disease having lung lesion successfully treated with humanized anti-interleukin-6 receptor antibody, Tocilizumab. J Korean Med Sci 2010;25:1364-7.

45. Electronic Medicines Compendium [Internet]. RoActemra $20 \mathrm{mg} / \mathrm{ml}$ concentrate for solution for infusion. Available from: https://www.medicines.org.uk/EMC/medicine/22311/SPC/RoA ctemra+20mg+ml+Concentrate+for+Solution+for+Infusion/\#

46. Srirangan S, Choy EH. The role of interleukin 6 in the pathophysiology of rheumatoid arthritis. Ther Adv Musculoskelet Dis 2010;2:247-56.

47. Luo P, Liu Y, Qiu L, et al. Tocilizumab treatment in COVID19: A single center experience. J Med Virol 2020;92:814-8. doi: 10.1002/jmv.25801

48. Xu X, Han M, Li T, et al. Effective treatment of severe Covid19 patients with tocilizumab. Proc Natl Acad Sci USA 2020;117:10970-5. doi: 10.1073/pnas.2005615117

49. Mair-Jenkins J, Campos M, Baillie JK. The effectiveness of convalescent plasma and hyperimmune immunoglobulins for the treatment of severe acute respiratory infections of viral etiology: a systematic review. J Infect Dis 2015;211:80-90.

50. Pandey S, Vyias G. Adverse effects of plasma transfusion. Trasfusion 2012;52:65-79.

51. Shen C, Wang Z, Zhao F, et al. Treatment of 5 critically Ill patients with COVID-19 With convalescent plasma. JAMA 2020; 323:1582-9. doi: 10.1001/jama.2020.4783

52. Ahn JY, Sohn Y, Lee SH, et al. Use of convalescent plasma therapy in two COVID-19 patients with acute respiratory distress syndrome in Korea. J Korean Med Sci 2020;35:e149. doi: 10.3346/jkms.2020.35.e149 
53. Winau F, Winau R. Emil von Behring and serum therapy. Microbes Infect 2002;4:185-8.

54. Casadevall A, Scharff MD. Serum therapy revisited: animal models of infection and development of passive antibody therapy. Antimicrob Agents Chemother 1994;38:1695-702.

55. Stiehm ER, Ashida E, Kim KS, et al. Intravenous immunoglobulins as therapeutic agents. Ann Intern Med 1987; 107:367-82.

56. Fischer J, Zanker K, van Griensven M, et al. The role of passive immunization in the age of SARS-Cov-2: an update. Eur J Med Res 2020;25:16. doi: 10.1186/s40001-020-00414-5
57. Zeng F, Dai C, Cai P, et al. A comparison study of SARS-CoV2 IgG antibody between male and female COVID-19 patients: A possible reason underlying different outcome between sex. J Med Virol 2020;92:2050-4. doi: 10.1002/jmv.25989

58. Stiehm ER. Adverse effects of human immunoglobulin therapy. Transfus Med Rev 2013;27:171-8. doi:1 0.1016/j. tmrv.2013.05.004

59. Bischof E, Wolfe J, Klein SL. Clinical trials for COVID-19 should include sex as a variable. J Clin Invest 2020. doi: 10.1172/JCI139306 